\title{
Wavelet boundary element methods Adaptivity and goal-oriented error estimation
}

Helmut Harbrecht and Manuela Moor

\begin{abstract}
This article is dedicated to the adaptive wavelet boundary element method. It computes an approximation to the unknown solution of the boundary integral equation under consideration with a rate $N_{\text {dof }}^{-s}$, whenever the solution can be approximated with this rate in the setting determined by the underlying wavelet basis. The computational cost scale linearly in the number $N_{\text {dof }}$ of degrees of freedom. Goal-oriented error estimation for evaluating linear output functionals of the solution is also considered. An algorithm is proposed that approximately evaluates a linear output functional with a rate $N_{\text {dof }}^{-(s+t)}$, whenever the primal solution can be approximated with a rate $N_{\text {dof }}^{-s}$ and the dual solution can be approximated with a rate $N_{\text {dof }}^{-t}$, while the cost still scale linearly in $N_{\text {dof }}$. Numerical results for an acoustic scattering problem and for the point evaluation of the potential in case of the Laplace equation are reported to validate and quantify the approach.
\end{abstract}

\section{Introduction}

Many mathematical models concerning for example field calculations, flow simulation, elasticity or visualization are based on operator equations with nonlocal operators, especially boundary integral operators. The discretization of such problems will then amount to a large system of linear equations with a dense system matrix. Thus, the numerical solution of such problems requires large amounts of time and computation capacities.

Helmut Harbrecht

Departement Mathematik und Informatik, Universität Basel, Spiegelgasse 1, 4051 Basel, Schweiz, e-mail: helmut.harbrecht@unibas.ch

Manuela Moor,

Universitätsspital Basel, Spitalstrasse 21/Petersgraben 4, 4051 Basel, Schweiz,

e-mail:manuela.moor@usb.ch 
To overcome this obstruction, several ideas for the efficient approximation of the discrete system have been developed in the last decades. Most prominent examples are the fast multipole method [27, 37], the panel clustering [29], the adaptive cross approximation [3, 4], or hierarchical matrices [28, 38], all of which are known to reduce the computational cost to be nearly linear or even linear. A further approach is the wavelet boundary element method $[7,12,31]$ which employs that the wavelets' vanishing moments lead, in combination with the fact that the kernels of integral operators become smoother when getting farther away from the diagonal, to a quasi-sparse system matrix. Since the number of relevant entries in the system matrix for maintaining the convergence rate of the underlying Galerkin method scales only linearly, wavelet matrix compression leads to a numerical algorithm that has linear cost.

Another issue to be addressed for the efficient discretization of boundary integral equations is the one of adaptivity. For non-smooth geometries or right-hand sides, it is necessary to be able to resolve specific parts of the geometry, while other parts could stay coarse. The adaptive wavelet boundary element method has been developed in [13, 26, 33], based on the ideas of related adaptive wavelet methods for local operators from $[9,10,25]$. Assume that the solution of the boundary integral equation to be solved is known and can be approximated with a rate $N_{\text {dof }}^{-s}$ in the setting determined by the underlying wavelet basis. Then, the adaptive wavelet boundary element method computes an approximation that converges with a rate $N_{\text {dof }}^{-s}$ at a cost expense that scales linearly with $N_{\text {dof. }}$. The method is hence computationally optimal. Although reliable error estimators for boundary integral operators exist, see e.g., [19], and optimal convergence of traditional boundary element discretizations have been proven, see, e.g., [21, 24], we are not aware of any other boundary element method which is optimal in this sense.

For many applications one is not interested in the unknown solution, but only in a continuous, linear output functional of it. Approximating this new quantity of interest instead is referred to as goal-oriented method. By considering only an output functional, one is able to perform the computation with much less degrees of freedom. This goal-oriented adaptivity has intensively been studied in the field of adaptive finite element methods, see e.g. $[2,5,6,14,18,22,36]$ and the references therein. Optimal convergence rates have been analyzed in [22, 36]. For goal-oriented adaptive boundary element methods, only few results can be found [1, 20, 22]. The combination of goal-oriented adaptivity and fast boundary element methods has, however, not been considered yet.

We will present a goal-oriented strategy for the adaptive wavelet boundary element method. The strategy is in accordance with [36] and separately minimizes the error of the primal problem and the error of the dual problem, respectively. One computes two index sets which indicate the possible refinement, one for the primal problem and one for the dual problem. By choosing the smaller index for refinement, the functional evaluation converges at a rate $N_{\text {dof }}^{-(s+t)}$, whenever the primal solution can be approximated at a rate $N_{\text {dof }}^{-s}$ and the dual solution can be approximated at a rate $N_{\text {dof }}^{-t}$. The advantage of using the adaptive wavelet boundary element method instead of a traditional boundary element method as in $[20,21,22]$ is that 
the computational cost of the algorithm scales linearly with respect to the number $N_{\text {dof }}$ of degrees of freedom.

We would like to mention at this point that the goal-oriented approach is not restricted to linear output functionals, but can also be extended to non-linear output functionals, see e.g. [2] and the references therein. However, for the sake of a thorough convergence analysis, we are considering only a linear output functional here.

The outline is as follows. In Section 2, we recall the adaptive wavelet boundary element method. Then, in Section 3, we propose the goal-oriented refinement strategy. Numerical results for an acoustic scattering problem and for point evaluations of the single layer potential in case of the Laplace equation are presented in Section 4. Finally, concluding remarks are stated in Section 5.

Throughout this article, in order to avoid the repeated use of generic but unspecified constants, we mean by $C \lesssim D$ that $C$ can be bounded by a multiple of $D$, independently of parameters which $C$ and $D$ may depend on. Obviously, $C \gtrsim D$ is defined as $D \lesssim C$, and $C \sim D$ as $C \lesssim D$ and $C \gtrsim D$.

\section{Adaptive wavelet methods for boundary integral equations}

\subsection{Problem formulation}

Let $\Omega \subset \mathbb{R}^{n+1}$ be a bounded domain with Lipschitz-smooth boundary $\Gamma=\partial \Omega$. Adaptive wavelet methods rely on an iterative solution method for the continuous boundary integral equation

$$
(\mathscr{A} u)(\boldsymbol{x})=\int_{\Gamma} k(\boldsymbol{x}, \boldsymbol{y}) u(\boldsymbol{y}) \mathrm{d} \sigma_{\boldsymbol{y}}=f(\boldsymbol{x}), \quad \boldsymbol{x} \in \Gamma,
$$

under consideration, expanded with respect to a wavelet basis. Here, $\mathscr{A}: H^{q}(\Gamma) \rightarrow$ $H^{-q}(\Gamma)$ denotes an elliptic, symmetric, and continuous boundary integral operator ${ }^{1}$ of order $2 q$ with standard kernel $k$, satisfying

$$
\left|\partial_{\widehat{\boldsymbol{x}}}^{\boldsymbol{\alpha}} \partial_{\widehat{\boldsymbol{y}}}^{\boldsymbol{\beta}} k(\widehat{\boldsymbol{x}}, \widehat{\boldsymbol{y}})\right| \leq c_{\boldsymbol{\alpha}, \boldsymbol{\beta}}\|\widehat{\boldsymbol{x}}-\widehat{\boldsymbol{y}}\|^{-(|\boldsymbol{\alpha}|+|\boldsymbol{\beta}|+n+2 q)}
$$

for all $\widehat{\boldsymbol{x}}, \widehat{\boldsymbol{y}} \in \Gamma$ with $\widehat{\boldsymbol{x}} \neq \hat{\boldsymbol{y}}$ provided that $|\boldsymbol{\alpha}|+|\boldsymbol{\beta}|+n+2 q \geq 0$, where the derivation has to be understood with respect to the surface coordinates. We should remark that the kernel of a boundary integral operator $\mathscr{A}$ of order $2 q$ is in general a standard kernel of order $2 q$, see, e.g., [34]. This holds especially true for the kernel function associated with the Laplace and the Helmholtz equation, the system of Navier-Lamé equations and the Stokes system.

${ }^{1}$ In accordance with [23], one might also consider a compact perturbation of an elliptic, symmetric, and continuous boundary integral operator. 
Having at hand a wavelet basis $\Psi$ for the underlying energy space $H^{q}(\Gamma)$, the Riesz property

$$
\|\Psi \boldsymbol{u}\|_{H^{q}(\Gamma)} \sim\|\boldsymbol{u}\| \text { for all } \boldsymbol{u} \in \ell^{2}
$$

constitutes an isomorphism between $u \in H^{q}(\Gamma)$ and $\boldsymbol{u} \in \ell^{2}$. Especially, (1) is equivalent to the well-posed problem of finding $u=\Psi \boldsymbol{u}$ such that the bi-infinite dimensional system of linear equations

$$
\boldsymbol{A} \boldsymbol{u}=\boldsymbol{f}, \quad \text { where } \boldsymbol{A}:=\langle\mathscr{A} \Psi, \Psi\rangle \quad \text { and } \quad \boldsymbol{f}:=\langle f, \Psi\rangle,
$$

holds. Suitable wavelet bases on surfaces have, for example, been constructed in $[15,16,30,32]$.

\subsection{Building blocks}

For the approximate solution of the infinite dimensional system (2) of linear equations, one has to perform matrix-vector multiplications by means of adaptive applications of the operator $\boldsymbol{A}$ under consideration. The building blocks COARSE, APPLY, RHS, and SOLVE, which are needed to design an adaptive algorithm of optimal complexity, have been identified in $[9,10]$.

In order to specify the properties of the building blocks, we shall introduce the approximation spaces

$$
\ell_{\tau}^{w}=\left\{\boldsymbol{u} \in \ell^{2}:|\boldsymbol{u}|_{\ell_{\tau}^{w}}:=\sup _{N \in \mathbb{N}} N^{-1 / \tau}\left|\gamma_{N}(\boldsymbol{u})\right|<\infty\right\}
$$

where $\gamma_{N}(\boldsymbol{u})$ denotes the $N$-th largest coefficient in modulus of the vector $\boldsymbol{u}$. It holds $\boldsymbol{u} \in \ell_{\tau}^{w}$ whenever $u=\Psi \boldsymbol{u}$ is contained in the Besov space $B_{\tau}^{q+n s}(\Gamma)$ with $\tau=(s+$ $1 / 2)^{-1}$, see, e.g., [17]. We require that the following statements hold true for $\bar{s}:=$ $(d-q) / n$, where $d$ denotes the order of polynomials which can be represented exactly by the wavelet discretization:

- Matrix-vector multiplication: $\boldsymbol{w}_{\Lambda^{\prime}}=\operatorname{APPLY}\left[\varepsilon, \boldsymbol{v}_{\Lambda}\right]$. Let $\varepsilon>0$ and let $\boldsymbol{v}_{\Lambda}$ consist of $|\Lambda|<\infty$ non-zero coefficients. Then, the output $\boldsymbol{w}_{\Lambda^{\prime}}$ satisfies

$$
\left\|A v_{\Lambda}-w_{\Lambda^{\prime}}\right\| \leq \varepsilon
$$

where, for any $s \leq \bar{s}$, only

$$
\left|\Lambda^{\prime}\right| \lesssim \varepsilon^{-1 / s}\left|\boldsymbol{v}_{\Lambda}\right|_{\ell_{\tau}^{w}}^{1 / s}
$$

coefficients are non-zero. The number of arithmetic operations and storage locations used by this call is bounded by some absolute multiple of

$$
\varepsilon^{-1 / s}\left|\boldsymbol{v}_{\Lambda}\right|_{\ell_{\mathcal{\tau}}^{w}}^{1 / s}+|\Lambda|+1
$$


- Approximation of the right-hand side: $f_{\Lambda}=\operatorname{RHS}[\varepsilon]$. Given $\varepsilon>0$, the output $\boldsymbol{f}_{\Lambda}$ satisfies

$$
\left\|f-f_{\Lambda}\right\| \leq \varepsilon
$$

and, for any $s \leq \bar{s}$, if $\boldsymbol{u} \in \ell_{\tau}^{w}$, then

$$
|\Lambda| \lesssim \varepsilon^{-1 / s}|\boldsymbol{u}|_{\ell_{\tau}^{w}}^{1 / s}
$$

The number of arithmetic operations and storage locations used by the call is bounded by some absolute multiple of

$$
\varepsilon^{-1 / s}|\boldsymbol{u}|_{\ell_{\tau}^{w}}^{1 / s}+1 .
$$

- Galerkin solver: $\boldsymbol{w}_{\Lambda}=\operatorname{SOLVE}[\varepsilon, \Lambda]$. This routine computes the solution $\boldsymbol{w}_{\Lambda}$ of the system of linear equations

$$
\boldsymbol{A}_{\Lambda} \boldsymbol{u}_{\Lambda}=\boldsymbol{f}_{\Lambda}, \quad \text { where } \quad \boldsymbol{A}_{\Lambda}:=\left\langle\mathscr{A} \Psi_{\Lambda}, \Psi_{\Lambda}\right\rangle \quad \text { and } \quad \boldsymbol{f}_{\Lambda}:=\left\langle f_{\Lambda}, \Psi_{\Lambda}\right\rangle,
$$

with accuracy

$$
\left\|u_{\Lambda}-w_{\Lambda}\right\| \leq \varepsilon
$$

The number of arithmetic operations and storage locations used by the call is bounded by some absolute multiple of

$$
\varepsilon^{-1 / s}\left|\boldsymbol{u}_{\Lambda}\right|_{\ell_{\tau}^{w}}^{1 / s}+|\Lambda|+1
$$

provided that $\boldsymbol{u}_{\Lambda} \in \ell_{\tau}^{w}$ for some $s \leq \bar{s}$.

- Coarsening routine: $\boldsymbol{w}_{\Lambda^{\prime}}=\operatorname{COARSE}\left[\boldsymbol{\theta}, \boldsymbol{w}_{\Lambda}\right]$. This routine produces for given $0 \leq$ $\theta \leq 1$ an index set $\Lambda^{\prime} \subset \Lambda$ such that the restriction $\boldsymbol{w}_{\Lambda^{\prime}}$ of the input vector $\boldsymbol{w}_{\Lambda}$ satisfies

$$
\left\|\boldsymbol{w}_{\Lambda^{\prime}}\right\| \leq \theta\left\|\boldsymbol{w}_{\Lambda}\right\|
$$

where $\left|\Lambda^{\prime}\right|$, up to some absolute constant factor, is minimal. The computational complexity is bounded by some absolute multiple of $|\Lambda|$.

Our particular implementation of these building blocks, satisfying all desired properties, is based on piecewise constant wavelets (i.e., $d=1$ ). In particular, we restrict the set of active wavelet functions to tree constraints which ensures the method's efficient implementation. Note that the coarsening routine for trees originates from [8], while the realization of RHS requires some a-priori knowledge on the right-hand side $f$. The matrix-vector multiplication APPLY has been constructed in $[13,33]$, see also [26] for related results. We mention that the main ingredient is wavelet matrix compression to sparsify the system matrix of the boundary integral operator under consideration, see Figure 1 for an illustration. Straightforward modifications of RHS and APPLY yield finally the routine SOLVE, cf. [25]. We skip all the details here and refer the reader to the cited references. 

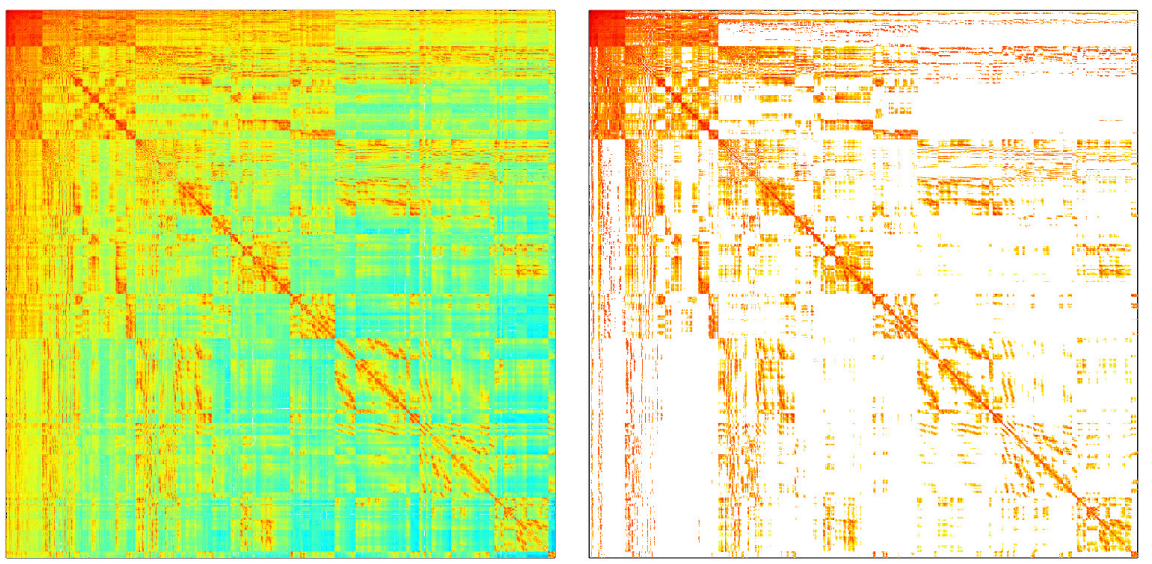

Fig. 1 Original matrix (left) and compressed matrix (right).

\subsection{An adaptive boundary element method}

The specific adaptive algorithm we use has been proposed in [25] and is similar to classical methods which consist of the following steps:

$$
\text { SOlVE } \rightarrow \text { Estimate } \rightarrow \text { MARK } \rightarrow \text { Refine }
$$

For a given (finite) index set $\Lambda \subset \ell^{2}$, we solve the Galerkin system (3) via $\boldsymbol{u}_{\Lambda}=$ $\operatorname{SOLVE}[\varepsilon, \Lambda]$ with appropriate accuracy $\varepsilon>0$. To estimate the (infinite) residual $\boldsymbol{r}:=\boldsymbol{f}-\boldsymbol{A} \boldsymbol{u}_{\Lambda}$, we compute an approximation $\boldsymbol{r}_{\Lambda^{\prime}}$ relative to a finite index set $\Lambda \subset$ $\Lambda^{\prime} \subset \ell^{2}$ such that

$$
(1-\omega)\left\|\boldsymbol{r}_{\Lambda^{\prime}}\right\| \leq\|\boldsymbol{r}\| \leq(1+\omega)\left\|\boldsymbol{r}_{\Lambda^{\prime}}\right\|
$$

for some fixed constant $0<\omega<1$. This can be realized by calling

$$
\boldsymbol{r}_{\Lambda^{\prime}}=\operatorname{ESTIMATE}\left[\boldsymbol{\delta}, \boldsymbol{u}_{\Lambda}\right]
$$

for an appropriately chosen initial precision $\delta>0$. The routine is defined in Algorithm, where the until-clause $2 \delta \leq \omega\left\|\boldsymbol{r}_{\Lambda^{\prime}}\right\|$ ensures that the iteration terminates when (4) holds since

$$
\|\boldsymbol{r}\| \geq\left\|\boldsymbol{r}_{\Lambda^{\prime}}\right\|-\left\|\boldsymbol{r}-\boldsymbol{r}_{\Lambda^{\prime}}\right\| \geq\left\|\boldsymbol{r}_{\Lambda^{\prime}}\right\|-2 \delta \geq(1-\omega)\left\|\boldsymbol{r}_{\Lambda^{\prime}}\right\|
$$

on the one hand and

$$
\|\boldsymbol{r}\| \leq\left\|\boldsymbol{r}_{\Lambda^{\prime}}\right\|+\left\|\boldsymbol{r}-\boldsymbol{r}_{\Lambda^{\prime}}\right\| \leq\left\|\boldsymbol{r}_{\Lambda^{\prime}}\right\|+2 \delta \leq(1+\omega)\left\|\boldsymbol{r}_{\Lambda^{\prime}}\right\|
$$

on the other hand.

The supporting index set $\Lambda^{\prime}$ of the approximate residual $\boldsymbol{r}_{\Lambda^{\prime}}$ enlarges the original index set $\Lambda$ such that the Galerkin solution with respect to $\Lambda^{\prime}$ would reduce the 


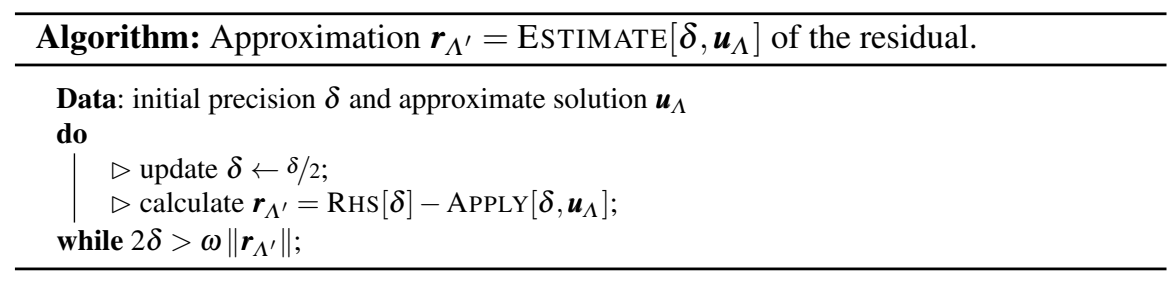

current error by a constant factor. Nonetheless, we need to coarsen the index set $\Lambda^{\prime}$ for controlling the complexity. This is done by calling the COARSE-routine

$$
\boldsymbol{r}_{\Lambda^{\prime \prime}}=\operatorname{COARSE}\left[\boldsymbol{\theta}, \boldsymbol{r}_{\Lambda^{\prime}}\right]
$$

for fixed $0<\theta<1$ sufficiently small. It combines the steps mark and refine since the new index set $\Lambda^{\prime \prime} \subset \Lambda^{\prime}$ still enlarges the original index set $\Lambda$, which corresponds to mesh refinement. Especially, it holds $\left\|\boldsymbol{r}_{\Lambda^{\prime \prime}}\right\| \sim\|\boldsymbol{r}\|$. Hence, the algorithm's convergence is guaranteed when repeating the procedure again with $\Lambda:=\Lambda^{\prime \prime}$. For all the details of the particular implementation, we refer the reader to [39].

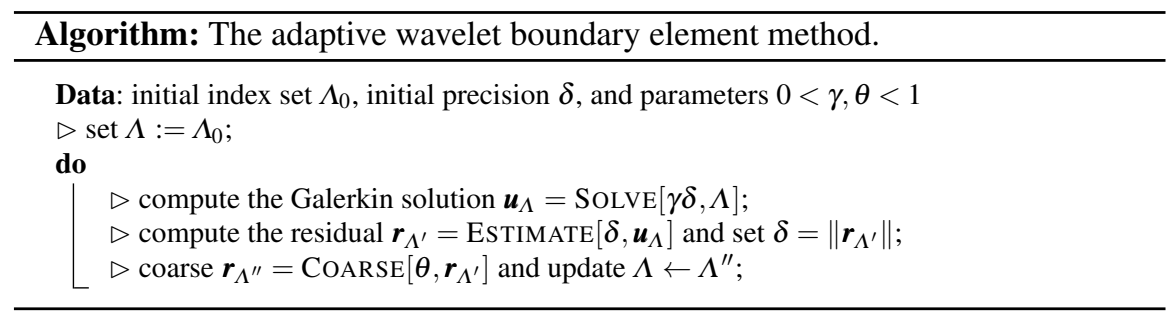

In accordance with [13, 25], having at hand the building blocks COARSE, APPLY, RHS, and SOLVE with the properties specified in Section 2.2, the following statement provides the optimality of Algorithm . Note that the hidden constant depends on the boundary integral operator under consideration, the wavelet basis and the choice of the parameters.

Theorem 1. Let $0<\gamma, \theta<1$ be sufficiently small parameters and let $\boldsymbol{u} \in \ell_{\tau}^{w}$ with $\tau=(s+1 / 2)^{-1}$ for some $s \leq \bar{s}$. Then, Algorithm computes iterates $\boldsymbol{u}_{\Lambda}$, which satisfy the error estimate

$$
\left\|\boldsymbol{u}-\boldsymbol{u}_{\Lambda}\right\| \lesssim|\Lambda|^{-s}
$$

at a computational expense that stays proportional to the number $|\Lambda|$ of degrees of freedom.

Proof. The assertion follows from the abstract theory presented in [25] for elliptic, symmetric, and continuous operators (cf. [25, Theorem 2.7]). The extension to compact perturbations of such operators is found in [24]. 


\section{Goal-oriented adaptivity}

We shall motivate the key idea of goal-oriented error estimation. To that end, let $a: V \times V \rightarrow \mathbb{R}$ be an elliptic and continuous bilinear form and $f \in V^{\prime}$. Consider the variational formulation

$$
\text { seek } u \in V \text { such that } a(u, v)=\langle f, v\rangle \text { for all } v \in V
$$

and the associated Galerkin scheme

$$
\text { seek } u_{\Lambda} \in V_{\Lambda} \text { such that } a\left(u_{\Lambda}, v_{\Lambda}\right)=\left\langle f, v_{\Lambda}\right\rangle \text { for all } v_{\Lambda} \in V_{\Lambda} \text {, }
$$

where $V_{\Lambda} \subset V$ denotes the trial space. At first glance, we obtain the error estimate

$$
\left|\langle g, u\rangle-\left\langle g, u_{\Lambda}\right\rangle\right|=\left|\left\langle g, u-u_{\Lambda}\right\rangle\right| \leq\|g\|_{V^{\prime}}\left\|u-u_{\Lambda}\right\|_{V}
$$

for the evaluation of an output functional $g \in V^{\prime}$. Nonetheless, by introducing the dual or adjoint solution

$$
\text { seek } z \in V \text { such that } a(v, z)=\langle g, v\rangle \text { for all } v \in V
$$

and observing Galerkin orthogonality, we conclude that

$$
\left|\langle g, u\rangle-\left\langle g, u_{\Lambda}\right\rangle\right|=\min _{z_{\Lambda} \in V_{\Lambda}}\left|a\left(u-u_{\Lambda}, z-z_{\Lambda}\right)\right| \lesssim \min _{z_{\Lambda} \in V_{\Lambda}}\left\|u-u_{\Lambda}\right\|_{V}\left\|z-z_{\Lambda}\right\|_{V}
$$

This fact greatly improves the error estimate and is exploited in the sequel.

Based on the adaptive wavelet boundary element method, proposed in the previous section, we can formulate a goal-oriented adaptive strategy to efficiently evaluate linear output functionals of the solution to the boundary integral equation (1) under consideration. As motivated above, we have now to synchronously approximate the solutions $\boldsymbol{u}, \boldsymbol{z} \in \ell^{2}$ of the two systems of linear equations,

$$
\boldsymbol{A} \boldsymbol{u}=\boldsymbol{f} \quad \text { and } \quad \boldsymbol{A}^{\top} \boldsymbol{z}=\boldsymbol{g},
$$

where $\boldsymbol{A}$ and $\boldsymbol{f}$ are defined as in (2) and $\boldsymbol{g}=\langle g, \Psi\rangle$ denotes the discretized output functional, where $\langle g, u\rangle=\boldsymbol{g}^{\top} \boldsymbol{u}$. We therefore modify the adaptive wavelet boundary element method from Algorithm as follows.

For a given finite index set $\Lambda$, the primal and dual systems of linear equations are solved with sufficient accuracy. This yields the approximations $\boldsymbol{u}_{\Lambda}$ and $z_{\Lambda}$ to the primal and dual solution of (5), respectively. We then call

$$
\boldsymbol{r}_{p, \Lambda_{p}}=\text { EsTIMATE }_{\text {primal }}\left[\boldsymbol{\delta}_{p}, \boldsymbol{u}_{\Lambda}\right] \text { and } \boldsymbol{r}_{d, \Lambda_{d}}=\operatorname{EsTIMATE}_{\mathrm{dual}}\left[\boldsymbol{\delta}_{d}, \boldsymbol{z}_{\Lambda}\right]
$$

which refer to the primal and dual versions of the routine ESTIMATE as outlined in Algorithm . The input parameters $\delta_{p}$ and $\delta_{d}$ are initialized at the beginning by a $\delta_{\text {init }}$ of our choice, and they are modified during the course of the algorithm as outlined in Algorithm . 
Next, we call

$$
\boldsymbol{r}_{p, \Lambda_{p}^{\prime}}=\operatorname{COARSE}\left[\boldsymbol{\theta}, \boldsymbol{r}_{p, \Lambda_{p}}\right] \quad \text { and } \quad \boldsymbol{r}_{d, \Lambda_{d}^{\prime}}=\operatorname{COARSE}\left[\boldsymbol{\theta}, \boldsymbol{r}_{d, \Lambda_{d}}\right]
$$

to compute appropriate refinements $\Lambda_{p}^{\prime}, \Lambda_{d}^{\prime} \supset \Lambda$ of the original index set $\Lambda$. Finally, we choose the smaller of the two index sets $\Lambda_{p}$ and $\Lambda_{d}$ to update the index set $\Lambda$ and restart the loop.

The aforementioned goal-oriented adaptive refinement strategy is summarized in Algorithm . In accordance with [20, 22, 36], we derive the following result on the goal-oriented wavelet boundary element method, provided that the building blocks COARSE, APPLY, RHS, and SOLVE satisfy the properties specified in Subsection 2.2.

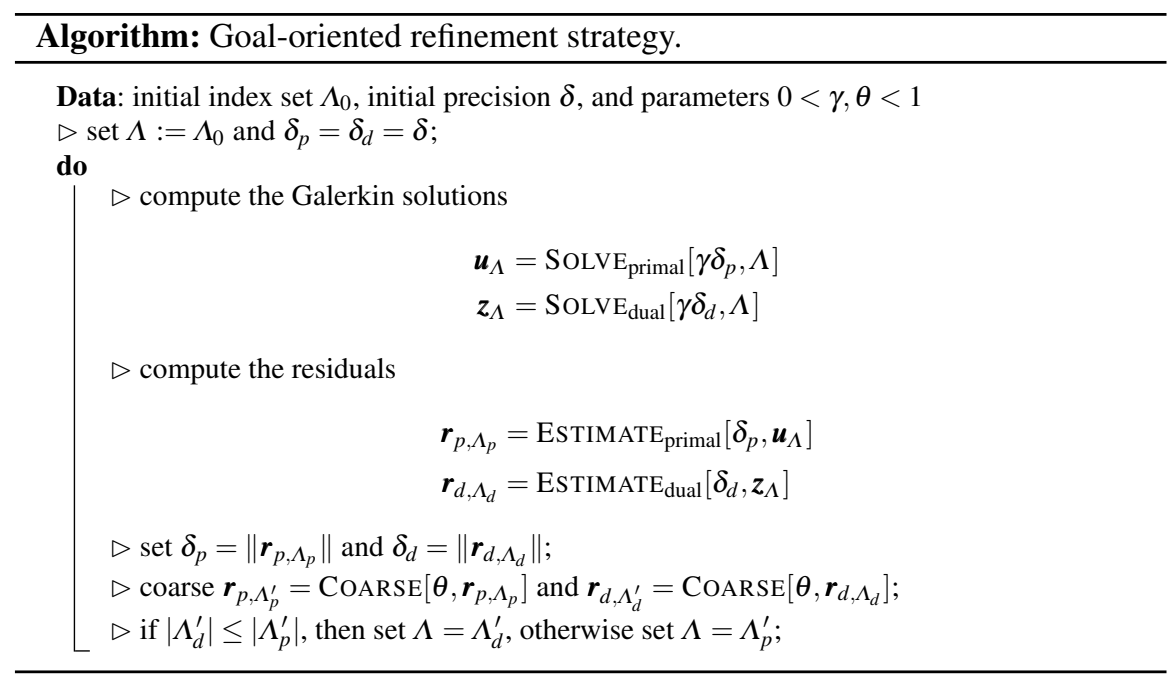

Proposition 1. Let $0<\gamma, \theta, \omega<1$ be sufficiently small parameters and let $\boldsymbol{u} \in \ell_{\tau_{p}}^{w}$ with $\tau_{p}=(s+1 / 2)^{-1}$ and $z \in \ell_{\tau_{d}}^{w}$ with $\tau_{d}=(t+1 / 2)^{-1}$ for some $s, t \leq \bar{s}$. Then, the approximation $\boldsymbol{u}_{\Lambda}$ of the primal solution $\boldsymbol{u}$ and the approximation $z_{\Lambda}$ of the dual solution $z$, computed by Algorithm, satisfy the error estimate

$$
\left\|\boldsymbol{u}-\boldsymbol{u}_{\Lambda}\right\|\left\|\boldsymbol{z}-\boldsymbol{z}_{\Lambda}\right\| \lesssim|\Lambda|^{-(s+t)} .
$$

The computational expense to compute these approximations scales proportional to the number $|\Lambda|$ of degrees of freedom.

Proof. By construction, the norms of the approximate primal residual $\boldsymbol{r}_{p, \Lambda_{p}}$ and the approximate dual residual $\boldsymbol{r}_{d, \Lambda_{d}}$ are always equivalent to the respective exact residual: 


$$
\begin{aligned}
& (1-\omega)\left\|\boldsymbol{r}_{p, \Lambda_{p}}\right\| \leq\left\|\boldsymbol{r}_{p}\right\| \leq(1+\omega)\left\|\boldsymbol{r}_{p, \Lambda_{p}}\right\|, \\
& (1-\omega)\left\|\boldsymbol{r}_{d, \Lambda_{d}}\right\| \leq\left\|\boldsymbol{r}_{d}\right\| \leq(1+\omega)\left\|\boldsymbol{r}_{d, \Lambda_{d}}\right\| .
\end{aligned}
$$

Therefore, we have

$$
\left\|\boldsymbol{u}-\boldsymbol{u}_{\Lambda}\right\|\left\|\boldsymbol{z}-\boldsymbol{z}_{\Lambda}\right\| \sim\left\|\boldsymbol{r}_{p, \Lambda_{p}}\right\|\left\|\boldsymbol{r}_{d, \Lambda_{d}}\right\| \sim \delta_{p} \delta_{d}
$$

Thus, $|\Lambda| \lesssim\left(\delta_{p} \delta_{d}\right)^{-1 /(s+t)}$ in accordance with [36, Theorem 5.5], which implies (6). The complexity bound is finally an immediate consequence of the optimality of the adaptive wavelet method.

\section{Numerical results}

\subsection{Scattering Problems}

We shall present numerical results for an acoustic scattering problem with different wavenumbers $\kappa \geq 1$. The choice of larger wavenumbers has a direct effect on the sparsity of the system matrix, as the compression parameters have to be proportionally increased with the wavenumber, see [35]. Therefore, the method cannot be expected to be robust with respect to the wavenumber.

Given a sound-soft scatterer $\Omega \subset \mathbb{R}^{3}$, we consider the solution $u$ of the exterior Helmholtz equation

$$
\Delta u+\kappa u=0 \text { in } \mathbb{R}^{3} \backslash \bar{\Omega}, \quad u=0 \text { on } \Gamma:=\partial \Omega .
$$

The function $u$ consists of an incident and a scattered wave, i.e. $u=u^{s}+u^{i}$, where in addition to (7) the scattered wave satisfies the Sommerfeld radiation condition

$$
\lim _{r \rightarrow \infty} r\left(\frac{\partial u^{s}}{\partial r}-i \kappa u^{s}\right)=0 \text { as } r=\|x\| \rightarrow \infty .
$$

The incident wave $u^{i}$ is known and is of the form $\exp (i \kappa \boldsymbol{d} \boldsymbol{x})$, where $\boldsymbol{d}$ denotes the direction (it holds $\|\boldsymbol{d}\|=1$ ), and the goal is to compute the scattered wave $u^{s}$. When $u^{s}$ is given, the solution $u$ to the Helmholtz equation (7) can be computed as well.

In order to find $u$, we use the direct ansatz

$$
u(\boldsymbol{x})=u^{i}(\boldsymbol{x})-\frac{1}{4 \pi} \int_{\Gamma} k(\boldsymbol{x}, \boldsymbol{y}) \frac{\partial u}{\partial \boldsymbol{n}}(\boldsymbol{y}) \mathrm{d} \sigma_{\boldsymbol{y}}, \quad \boldsymbol{x} \in \mathbb{R}^{3} \backslash \overline{\boldsymbol{\Omega}} .
$$

Here, $k(\cdot, \cdot)$ denotes the fundamental solution to the Helmholtz equation, given by

$$
k(\boldsymbol{x}, \boldsymbol{y})=\frac{1}{4 \pi} \frac{e^{i \kappa\|\boldsymbol{x}-\boldsymbol{y}\|}}{\|\boldsymbol{x}-\boldsymbol{y}\|}
$$


In accordance with [11], the unknown Neumann data $\frac{\partial u}{\partial n}$ are obtained by solving the Fredholm boundary integral equation of the second kind (i.e., $q=0$ )

$$
\left(\frac{1}{2}+\mathscr{D}^{\top}-i \eta \mathscr{S}\right) \frac{\partial u}{\partial \boldsymbol{n}}=\frac{\partial u^{i}}{\partial \boldsymbol{n}}-i \eta u^{i} \quad \text { on } \Gamma
$$

where $\mathscr{S}$ and $\mathscr{D}$ are the acoustic single and double layer operators, respectively,

$$
(\mathscr{S} v)(\mathbf{x})=\int_{\Gamma} k(\boldsymbol{x}, \boldsymbol{y}) v(\boldsymbol{y}) \mathrm{d} \sigma_{\boldsymbol{y}}, \quad(\mathscr{D} v)(\mathbf{x})=\int_{\Gamma} \frac{\partial}{\partial \boldsymbol{n}_{\boldsymbol{y}}} k(\boldsymbol{x}, \boldsymbol{y}) v(\boldsymbol{y}) \mathrm{d} \sigma_{\boldsymbol{y}}
$$

and $\eta>0$ is a parameter which is usually chosen proportional to $\kappa$, see [11] for example.

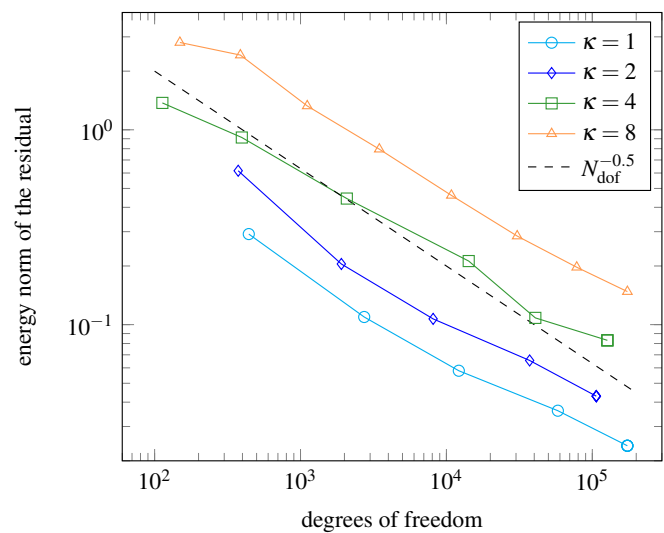

Fig. 2 Energy norm of the residual for the adaptive wavelet method in dependance of the wavenumbers.

For our numerical computations, we will solve the scattering problem by using the boundary integral equation (9) for the wavenumbers $\kappa=1,2,4$, and 8 . For the adaptive algorithm, $\omega=0.5, \theta=0.9$, and $\gamma=10^{-2}$ were chosen. The arising system of linear equations is solved by means of the GMRES method with diagonal scaling, where the approximate solution from the previous step is used as initial guess and the system is solved up to a relative precision $10^{-5}$ (we refer the reader to [39] for parameter studies and details of the current implementation).

Having the approximate solution at hand, we have given the Neumann data which can be used to evaluate $u(\boldsymbol{x})$ according to the ansatz (8). As scatterer $\Omega$, we consider a drilled cube as seen in Figures 4-7. The incident wave is chosen to travel into the direction of $(1,1,0)$.

Figure 2 shows the convergence history of the estimated norm of the residual for each different value of $\kappa$. We observe a rate of convergence of approximately $N_{\text {dof }}^{-0.5}$, independently of the chosen $\kappa$, while the norm of the residual increases as the 


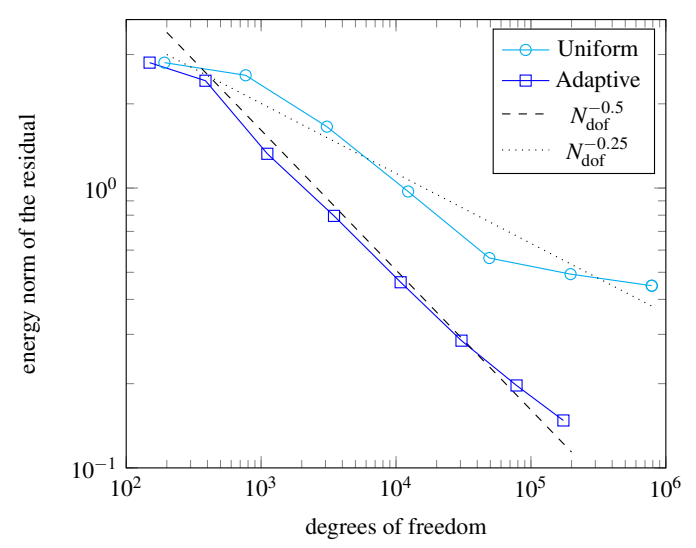

Fig. 3 Norm of the residual for adaptive refinement and for uniform refinement.

wavenumber increases. A comparison of the rate of convergence in case of adaptive and uniform refinement is found in Figure 3 for the wavenumber $\kappa=8$. We observe approximately the rate of convergence $N_{\mathrm{dof}}^{-0.25}$ in case of uniform refinement, which is only half the rate as in case of adaptive refinement.

For the visualization of the solution, we compute the total field $u(x)$ and the scattered field $u^{s}(\boldsymbol{x})$ in the area $E=\left\{\left(x_{1}, x_{2}, x_{3}\right): x_{3}=0\right.$ and $\left.x_{1}, x_{2} \in[-2.5,2.5]\right\}$. This plane intersects the drilled cube, such that we can illustrate the pattern which is produced by the scattered wave. In addition to the plane, where $u^{s}(\boldsymbol{x})$ and $u(\boldsymbol{x})$ are evaluated, we also draw the scatterer in the pictures. In particular, we draw the refinement of the scatterer's surface, where a cluster of wavelets appears in a darker colour first. By looking more closely at the corners and edges of the geometry, we see a lighter colouring, which again indicates even a stronger refinement.
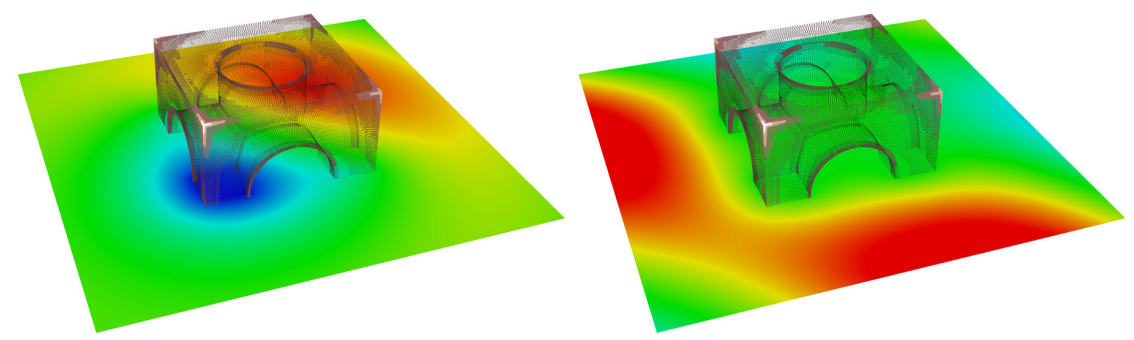

Fig. 4 Scattered field (left) and total field (right) for $\kappa=1$.

In Figure 4, we see the scene for $\kappa=1$. The top corner of the square is the point $(-2.5,-2.5)$, which means that the harmonic wave is travelling upwards. In the left plot of Figure 4, the scattered field $u^{s}(\boldsymbol{x})$ is seen and, in the right plot of Figure 4, 
the total field $u(\boldsymbol{x})$ is seen. For $\kappa=1$, we do not observe yet an interesting scattering pattern, as the wavenumber is too small. On the other hand, we already observe that the adaptive wavelet boundary element method refines towards the edges and the vertices of the geometry. This behaviour is expected, since we solve the scattering problem for the direct formulation involving the Neumann data, which admit a singularity at the non-smooth parts of the geometry. In particular, we observe a refinement on the edges which are illuminated, i.e. the edges which face the incoming wave. On the edges which are at the back of the geometry refinement, the refinement is not that strong.
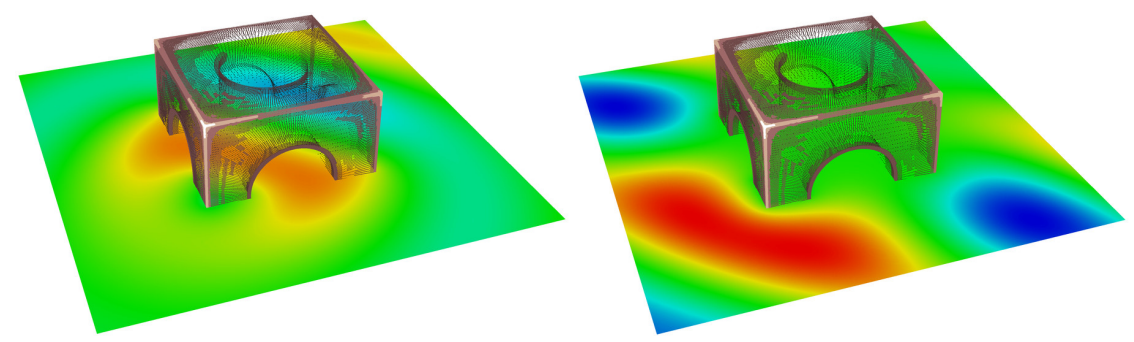

Fig. 5 Scattered field (left) and total field (right) for $\kappa=2$.

In Figure 5, we visualize the scattered field and the total field for $\kappa=2$. We observe that the wavenumber $\kappa=2$ is still too small to have a noticeable scattering pattern. Also, we observe again the refinement along the illuminated edges in reference to the incoming wave.
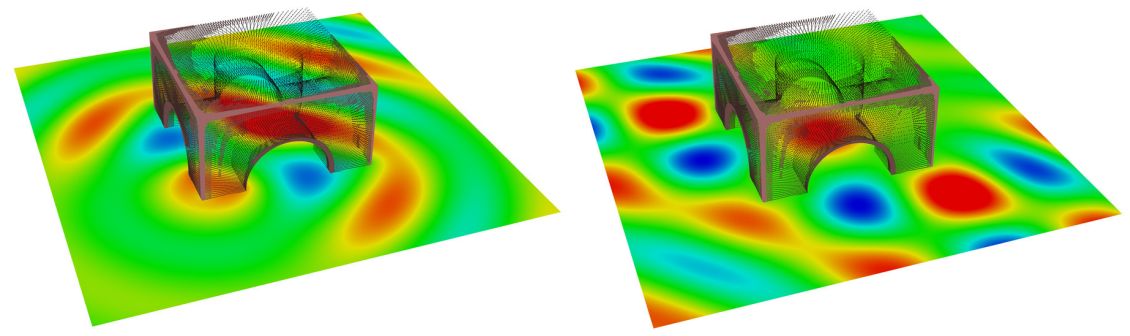

Fig. 6 Scattered field (left) and total field (right) for $\kappa=4$.

Figure 6 contains the scattered field (left) and total field (right) for the wavenumber $\kappa=4$. Here, we observe that the wavenumber is chosen just large enough, such that for the first time the wave can enter the inner part of the drilled cube. We observe again the refinement towards the edges and vertices facing the incoming wave, with less refinement in those parts of the geometry which lie on the back of the cube. 

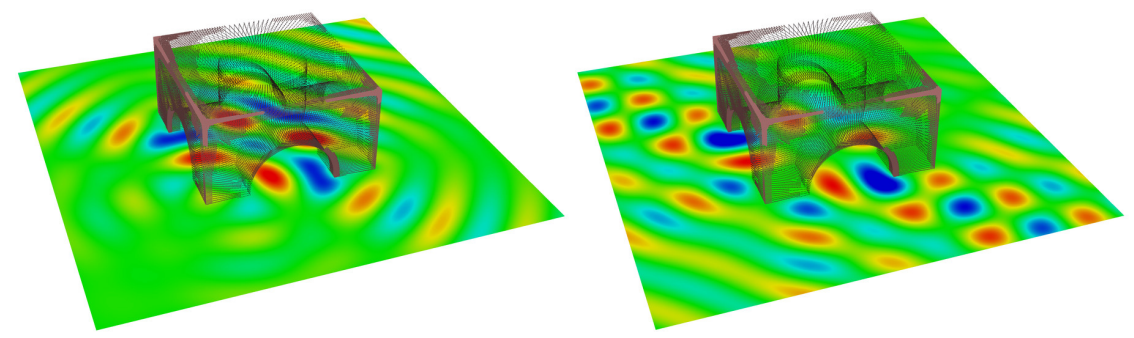

Fig. 7 Scattered field (left) and total field (right) for $\kappa=8$.

In Figure 7, we draw the scattered field (left) and the total field (right) for the wavenumber $\kappa=8$. This wavenumber is large enough in order to produce a beautiful scattering pattern. Especially, we see that the wave can travel through the inner part of the drilled cube. We observe again the refinement towards the edges and vertices with more refinement of those parts of the drilled cube which are illuminated by the incoming wave.

We conclude that the adaptive wavelet algorithm produces excellent results for all chosen wavenumbers $\kappa$. Adaptivity pays off especially for the direct ansatz, where the refinement towards the edges and vertices can clearly be observed. To achieve a similar accuracy with uniform mesh refinement, one would need much more degrees of freedom. This would not only take more time to compute, but may not be feasible any more as far as memory consumption is concerned.

\subsection{Laplace equation solved by the single layer operator}

Let us present numerical results in order to verify and quantify the goal-oriented adaptive wavelet boundary element method. To this end, consider the Laplace equation

$$
\Delta U=0 \text { in } \Omega, \quad U=f \text { on } \Gamma,
$$

solved inside a bounded domain $\Omega$ with boundary $\Gamma=\partial \Omega$. We convert this problem to a boundary integral equation by making the ansatz

$$
U=\int_{\Gamma} \frac{u(\boldsymbol{y})}{\|\cdot-\boldsymbol{y}\|} \mathrm{d} \sigma_{\boldsymbol{y}} \text { in } \Omega
$$

for the unknown density $u \in H^{-1 / 2}(\Gamma)$. Observing that this single layer potential is continuous across the boundary $\Gamma$, we arrive at the Fredholm integral equation of the first kind

$$
\mathscr{A} u=\int_{\Gamma} \frac{u(\boldsymbol{y})}{\|\cdot-\boldsymbol{y}\|} \mathrm{d} \sigma_{\boldsymbol{y}}=f \text { on } \Gamma
$$


for the single layer operator $\mathscr{A}: H^{-1 / 2}(\Gamma) \rightarrow H^{1 / 2}(\Gamma)$. Given an evaluation point $\boldsymbol{x} \in \Omega$, the potential $U(\boldsymbol{x})$ is computed in accordance with (11) by

$$
g(u)=\int_{\Gamma} \frac{u(\boldsymbol{y})}{\|\boldsymbol{x}-\boldsymbol{y}\|} \mathrm{d} \sigma_{\boldsymbol{y}} .
$$

This potential evaluation corresponds to the application of a continuous linear functional to the density $u$. Notice that the integrand becomes weakly singular as $\boldsymbol{x} \in \Omega$ approaches the boundary $\Gamma$.

For the following computations, we consider the Fichera vertex $(0,1)^{3} \backslash(0,0.5]^{3}$ as domain of interest. The restriction $f=\left.p\right|_{\Gamma}$ of the harmonic polynomial $p(\boldsymbol{x})=$ $4 x_{1}^{2}-3 x_{2}^{2}-x_{3}^{2}$ is chosen as Dirichlet data for the primal problem, which implies that the solution $U$ of the Laplace equation (10) coincides with the polynomial $p$. For given, fixed $\boldsymbol{x} \in \Omega$, we shall apply the goal-oriented adaptive wavelet boundary element method to evaluate the output functional $g(u)$, given by equation (12). After each iteration of the adaptive algorithm, we compute an approximation $g\left(u_{\Lambda}\right)$ via the scalar product $\boldsymbol{g}_{\Lambda}^{\top} \boldsymbol{u}_{\Lambda}$. We then evaluate the potential error $\left\|p(\boldsymbol{x})-\boldsymbol{g}_{\Lambda}^{\top} \boldsymbol{u}_{\Lambda}\right\|$ with $p(\boldsymbol{x})$ being the analytic solution of the problem under consideration.

\subsubsection{First example}

We choose the evaluation point for the functional (12) as $\boldsymbol{x}=(0.25,0.25,0.9)$. This point is located inside Fichera's vertex but close to the top boundary. Moreover, we have chosen the coarsening constant $\theta=0.5$.

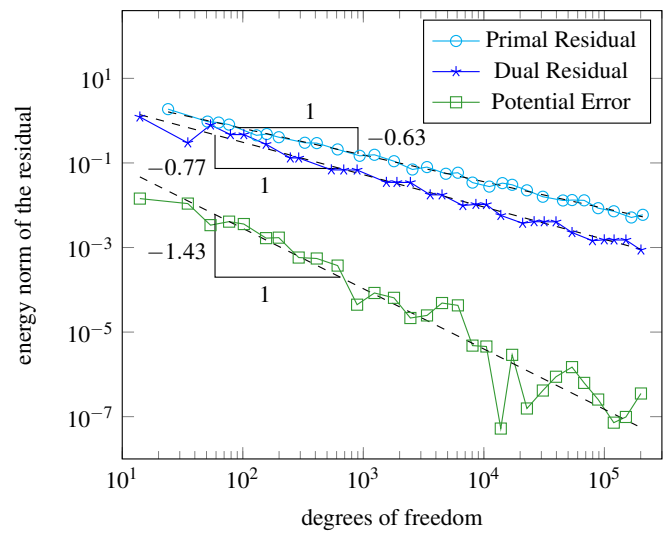

Fig. 8 Norm of the primal residual, norm of the dual residual and potential error versus the number of degrees of freedom in case of the evaluation point $\boldsymbol{x}=(0.25,0.25,0.9)$.

Figure 8 shows the convergence histories of the primal residual, the dual residual and the potential error of the goal-oriented adaptive wavelet boundary element 
method. We observe that the primal residual has a rate of convergence of $N_{\text {dof }}^{-0.63}$. Whereas, we notice that the dual residual seems to have a rate of convergence of approximately $N_{\mathrm{dof}}^{-0.75}$, which is significantly better than the rate of convergence for the primal residual. The potential error has a rate of convergence of approximately $N_{\text {dof }}^{-1.43}$, which indeed coincides with the sum of the rates of convergence for the primal and the dual residual.

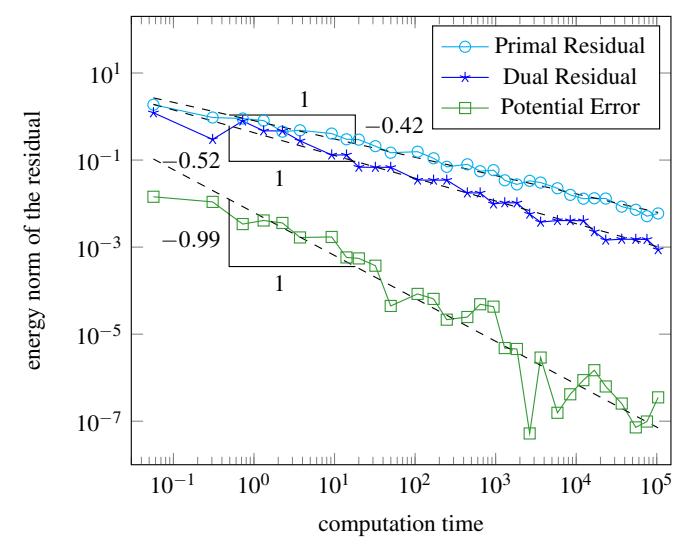

Fig. 9 Norm of the primal residual, norm of the dual residual, and potential error versus computation time in case of the evaluation point $\boldsymbol{x}=(0.25,0.25,0.9)$.

In Figure 9, we plot the ratios of the primal residual, the dual residual, and the potential error versus the computation time. It turns out that the computational complexity of the current implementation does not scale linearly but much better than quadratically. Therefore, it does clearly pay off to employ a fast boundary element method. Especially, to compute the solution to a boundary integral equation with more than 200,000 degrees of freedom would have not been possible without matrix compression.

We should also visualize the refinement which is produced by the adaptive algorithm. In Figure 10, we have visualized the refinement. Since we would not be able to see the refinement by drawing the grid, this picture was produced in the following way: After the code terminated, we assigned to each active wavelet a point in the center of its support which is weighted with 2 to the power of the wavelet's level, achieving that a small wavelet gets assigned a large value. The picture below is thus to be interpreted as: The lighter the colour, the finer are the elements in this area. We observe in Figure 10 that the mesh refinement takes place on the top of the Fichera vertex, near from where the point $\boldsymbol{x}=(0.25,0.25,0.9)$ is located. Also, the algorithm refines towards the edges and vertices of Fichera's vertex. 


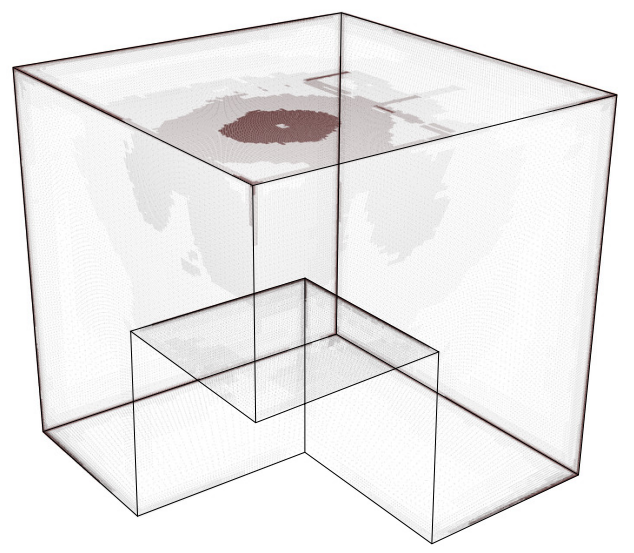

Fig. 10 Adaptive mesh refinement in case of the evaluation point $(0.25,0.25,0.9)$.

\subsubsection{Second example}

For the second example, we move the evaluation point more closely to the boundary, namely we set $\boldsymbol{x}=(0.25,0.25,0.95)$, and perform our computations again.

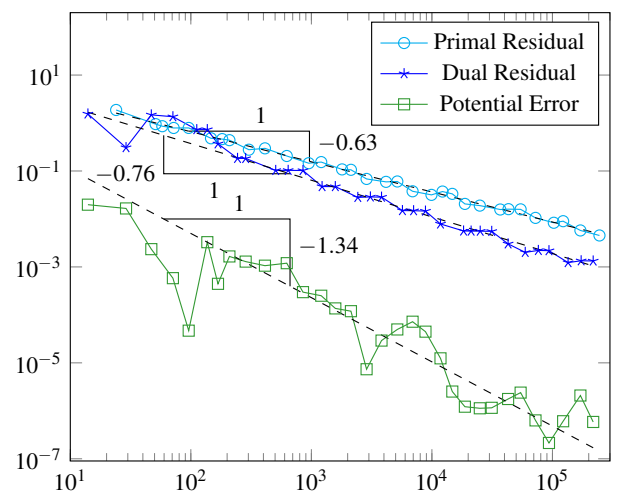

Fig. 11 Norm of the primal residual, norm of the dual residual, and potential error versus the number of degrees of freedom in case of the evaluation point $\boldsymbol{x}=(0.25,0.25,0.95)$.

In Figure 11, we visualize the convergence histories for the primal residual, the dual residual, and the potential error versus the number of degrees of freedom in a log-log scale. We observe that the primal residual and the dual residual show a rate of convergence of $N_{\text {dof }}^{-0.63}$ and $N_{\text {dof }}^{-0.76}$. For the potential error, we observe a rate of convergence of $N_{\mathrm{dof}}^{-1.34}$. This is slightly less than the rate of convergence of the potential error for the evaluation point $\boldsymbol{x}=(0.25,0.25,0.9)$. The computing time 
of the adaptive algorithm does not scale linearly in the number $N$ of degrees of freedom, compare Figure 12, where the accuracy versus computing times is plotted and the rates are slightly worse than those found in Figure 11. Nonetheless, we like to repeat that the scaling is much better than a quadratic scaling, which is required by the traditional boundary element method.

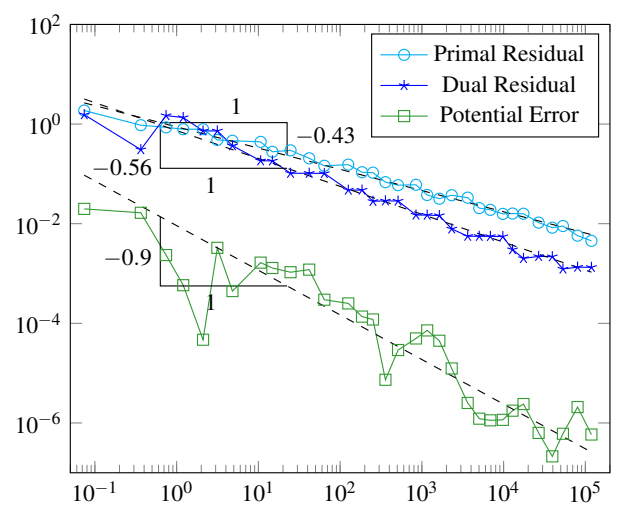

Fig. 12 Norm of the primal residual, norm of the dual residual, and potential error versus computation time in case of the evaluation point $\boldsymbol{x}=(0.25,0.25,0.95)$.

To conclude our tests, we finally visualize the mesh refinement produced by the adaptive algorithm in Figure 13. It is again refined towards the edges and vertices of the geometry and towards the point $\boldsymbol{x}=(0.25,0.25,0.95)$. If we compare the refinement on the top of the domain with the refinement from the previous example, we notice that the refinement is slightly more localized here, cf. Figure 10.

\section{Conclusion}

In the present article, we presented the adaptive wavelet boundary element method for the rapid solution of boundary integral equations. A goal-oriented strategy for the evaluation of linear output functionals has been proposed as well. The algorithms have been validated and quantified by numerical examples for an acoustic scattering problem and for the point evaluation of the potential in case of the single layer operator for the Laplace equation on Fichera's vertex.

Acknowledgements This research has been supported by the Swiss National Science Foundation (SNSF) through the DACH-project "BIOTOP: Adaptive Wavelet and Frame Techniques for Acoustic BEM". 


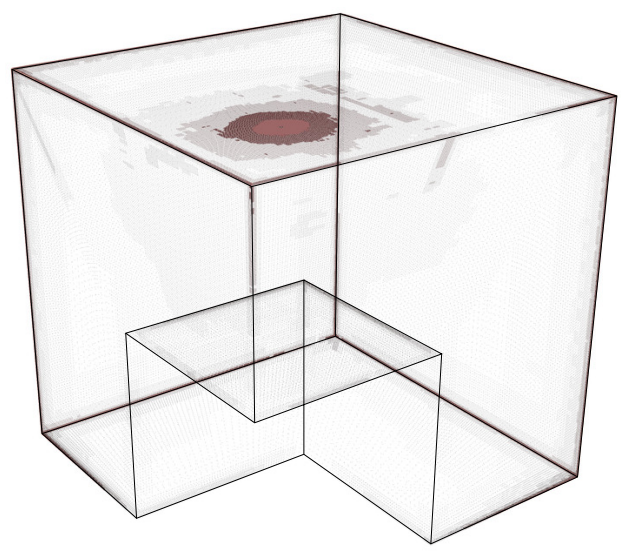

Fig. 13 Adaptive mesh refinement in case of the evaluation point $(0.25,0.25,0.95)$.

\section{References}

1. H. Bakry. A goal-oriented a posteriori error estimate for the oscillating single layer integral equation. Appl. Math. Lett., 69:133-137, 2017.

2. W. Bangerth and R. Rannacher. Adaptive finite element method for differential equations. Lectures Math. ETH Zürich. Birkhäuser, Basel, 2003.

3. M. Bebendorf. Approximation of boundary element matrices. Numer. Math., 86:565-589, 2000.

4. M. Bebendorf and S. Rjasanow. Adaptive low-rank approximation of collocation matrices. Computing, 70:1-24, 2003.

5. R. Becker, E. Estecahandy, and D. Trujillo. Weighted marking for goal-oriented adaptive finite element methods. SIAM J. Numer. Anal., 49(6):2451-2469, 2011.

6. R. Becker and R. Rannacher. An optimal control approach to a posteriori error estimation in finite element methods. Acta Numerica, 10:1-102, 2001.

7. G. Beylkin, R. Coifman, and V. Rokhlin. The fast wavelet transform and numerical algorithms. Comm. Pure and Appl. Math., 44:141-183, 1991.

8. P. Binev and R. DeVore. Fast computation in adaptive tree approximation. Numer. Math., 97:193-217, 2004.

9. A. Cohen, W. Dahmen, and R. DeVore. Adaptive wavelet methods for elliptic operator equations. Convergence rates. Math. Comput., 70:27-75, 2001.

10. A. Cohen, W. Dahmen, and R. DeVore. Adaptive wavelet methods II. Beyond the elliptic case. Found. Comput. Math., 2:203-245, 2002.

11. D. Colton and R. Kress. Integral equation methods in scattering theory. John Wiley \& Sons, New York, 1983

12. W. Dahmen, H. Harbrecht, and R. Schneider. Compression techniques for boundary integral equations. Asymptotically optimal complexity estimates. SIAM J. Numer. Anal., 43(6):22512271, 2006.

13. W. Dahmen, H. Harbrecht, and R. Schneider. Adaptive methods for boundary integral equations. Complexity and convergence estimates. Math. Comput., 76:1243-1274, 2007.

14. W. Dahmen, A. Kunoth, and J. Vorloeper. Convergence of adaptive wavelet methods for goal-oriented error estimation. In A. Bermudez de Castro, D. Gomez, P. Quintely, and P. Salgado, editors, Numerical mathematics and advanced applications, pages 39-61, Berlin, 2006. Springer. 
15. W. Dahmen and R. Schneider. Composite wavelet bases for operator equations. Math. Comput., 68:1533-1567, 1999.

16. W. Dahmen and R. Schneider. Wavelets on manifolds I. Construction and domain decomposition. Math. Anal., 31:184-230, 1999.

17. Ronald A. DeVore. Nonlinear approximation. Acta Numer., 7:51-150, 1998.

18. K. Eriksson, D. Estep, P. Hansbo, and C. Johnson. Introduction to adaptive methods for differential equations. Acta Numerica, 4:105-158, 1995.

19. B. Faermann. Localization of the Aronszajn-Slobodeckij norm and application to adaptive boundary element methods. Part II: The three-dimensional case. Numer. Math., 92(3):467499, 2002.

20. M. Feischl, G. Gantner, A. Haberl, D. Praetorius, and T. Führer. Adaptive boundary element methods for optimal convergence of point errors. Numer. Math., 132(3):541-567, 2016.

21. M. Feischl, M. Karkulik, J.M. Melenk, and D. Praetorius. Quasi-optimal convergence rate for an adaptive boundary element method. SIAM J. Numer. Anal., 51(2):1327-1348, 2013.

22. M. Feischl, D. Praetorius, and K.G. van der Zee. An abstract analysis of optimal goal-oriented adaptivity. SIAM J. Numer. Anal., 54(3):1423-1448, 2016.

23. T. Gantumur. An optimal adaptive wavelet method for nonsymmetric and indefinite elliptic problems. J. Comput. Appl. Math., 211(1):90-102, 2008.

24. T. Gantumur. Adaptive boundary element methods with convergence rates. Numer. Math., 124:471-516, 2013.

25. T. Gantumur, H. Harbrecht, and R. Stevenson. An optimal adaptive wavelet method for elliptic equations without coarsening. Math. Comput., 76:615-629, 2007.

26. T. Gantumur and R. Stevenson. Computation of singular integral operators in wavelet coordinates. Computing, 76:77-107, 2006.

27. L. Greengard and V. Rokhlin. A fast algorithm for particle simulation. J. Comput. Phys., 73:325-348, 1987.

28. W. Hackbusch. A sparse matrix arithmetic based on $\mathscr{H}$-matrices. Part I: Introduction to $\mathscr{H}$ matrices. Computing, 64:89-108, 1999.

29. W. Hackbusch and Z.P. Nowak. On the fast matrix multiplication in the boundary element method by panel clustering. Numer. Math., 54:463-491, 1989.

30. H. Harbrecht and R. Schneider. Biorthogonal wavelet bases for the boundary element method. Math. Nachr., 269-270:167-188, 2004.

31. H. Harbrecht and R. Schneider. Wavelet Galerkin schemes for boundary integral equations. implementation and quadrature. SIAM J. Sci. Comput., 27(4):1347-1370, 2006.

32. H. Harbrecht and R. Stevenson. Wavelets with patchwise cancellation properties. Math. Comput., 75(256): 1871-1889, 2006.

33. H. Harbrecht and M. Utzinger. On adaptive wavelet boundary element methods. J. Comput. Math., 36(1):90-109, 2018.

34. G.C. Hsiao and W.L. Wendland. Boundary integral equations, volume 164 of Applied Mathematical Sciences. Springer, Berlin, 2008.

35. D. Huybrechs, J. Simoens, and S. Vandevalle. A note on wave number dependence of wavelet matrix comression for integral equations with oscillatory kernel. J. Comput. Appl. Math., 172:233-246, 2004.

36. M.S. Mommer and R.P. Stevenson. A goal-oriented adaptive finite element method with convergence rates. SIAM J. Numer. Anal., 47(2):861-886, 2009.

37. V. Rokhlin. Rapid solution of integral equations of classical potential theory. J. Comput. Phys., 60:187-207, 1985.

38. E.E. Tyrtyshnikov. Mosaic sceleton approximation. Calcolo, 33:47-57, 1996

39. M. Utzinger. An Adaptive Wavelet Method for the Solution of Boundary Integral Equations in Three Dimensions. PhD thesis, Universität Basel, Switzerland, 2016. 